\title{
Zinc finger protein ZFP36L1 promotes osteoblastic differentiation but represses adipogenic differentiation of mouse multipotent cells
}

\author{
Kuo-Yun Tseng ${ }^{1}$, Yi-Hsuan Chen ${ }^{1}$ and Shankung Lin ${ }^{1,2}$ \\ ${ }^{1}$ Institute of Cellular and System Medicine, National Health Research Institutes, Zhunan Town, Miaoli, Taiwan, Republic of \\ China \\ ${ }^{2}$ Graduate Institute of Basic Medical Science, China Medical University, Taichung, Taiwan, Republic of China \\ Correspondence to: Shankung Lin, email: shankung@nhri.org.tw
}

Keywords: ZFP36L 1; osteoporosis; aging-related bone loss; osteoblastogenesis; adipogenesis; Gerotarget

Received: June 30, 2016

Accepted: November 09, 2016

Published: February 09, 2017

\section{ABSTRACT}

Zinc finger protein 36, C3H type-like 1 (ZFP36L1) is a member of the tristetraprolin (TTP) family and its role in the aging-related bone loss is currently unknown. We present evidence that ZFP36L1 expression in rat femurs and bone marrow mesenchymal stem cells (bmMSCs) was down-regulated with aging. ZFP36L1 knockdown decreased osteoblastic differentiation of MC3T3-E1 and C3H10T1/2 cells, and increased adipogenic differentiation of 3T3-L1 and C3H1OT1/2 cells, whereas ZFP36L1 overexpression did the opposite. The finding that ZFP36L1 overexpression enhanced osteoblastic and repressed adipogenic differentiation was also corroborated by ex vivo experiments. Troglitazone prevented ZFP36L1 from inhibiting adipogenic differentiation, suggesting the significance of PPARY2 repression in ZFP36L1's inhibitory effect on adipogenic differentiation. ZFP36L1 overexpression repressed the expression of Ppary 2 mRNA, but not the PPARY promoter activity. Biotin pulldown and electrophoretic mobility-shift assays suggested that ZFP36L1 might interact with endogenous Ppary2 mRNA by binding to its 3'UTR. The ZFP36L1-containing ribonucleoprotein complexes of ZFP36L1-overexpressing cells contained less Ppary2 mRNA than those of control cells. In a luciferase reporter construct, replacement of the SV40 poly(A) fragment by the 3'UTR of Ppary2 mRNA reduced the expression of luciferase transcripts in ZFP36L1-overexpressing cells. Examination of the kinetic expression of Ppary2 mRNA after transcriptional blockage showed that ZFP36L1 might enhance the degradation of the transcripts. Together, these data imply that ZFP36L1 overexpression might repress adipogenesis at least by down-regulating PPARY2 expression through post-transcriptional mechanisms. Thus, our findings support the notion that decrease of ZFP36L1 expression in bmMSCs with aging might contribute to the aging-related bone loss.

\section{INTRODUCTION}

Aging is associated with degeneration in many organs including bone. Aging bones exhibit reduced bone quality and bone mineral density, which decreases bone strength and renders the elderly prone to have fracture fall. It has been a worldwide task to search for remedies to counteract the aging-related bone deficit. Histological examinations on young and aging bones indicate a difference in the cell composition between the marrows of young and aging bones; aging bones contain more adipocytes but less osteoblasts than young bones $[1,2]$. Such a difference could be attributed to the increased adipogenic potential and decreased osteoblastic potential of bone marrow mesenchymal stem cells (bmMSCs), as suggested by the finding that after marrow ablation, bone marrows of aged rats are more adipogenic and less osteoblastogenic than those of young rats [3]. These observations indicate the decrease of osteoblast production as a significant contributor to bone loss in aging, and 
imply the existence of regulators in promoting adipogenic potential but suppressing osteoblatic potential of bmMSCs as a function of aging. Indeed, a transcription factor known as zinc finger factor 521 has recently been proposed as a potential regulator [4]. Therefore, to elucidate the mechanisms underlying the aging-related bone loss, it would be necessary to identify these regulators.

Zinc finger protein 36, C3H type-like 1 (ZFP36L1) is a member of the tristetraprolin (TTP) family. In rodents, TTP family comprises four members, including TTP, ZFP36L1, ZFP36L2, and ZFP36L3, whereas human TTP family comprises three members (TTP, ZFP36L1, and ZFP36L2) [5, 6]. TTP family members all contain tandem $\mathrm{CCCH}$ zinc fingers, which are responsible for RNAbinding. TTP family members can bind to the adenosine and uridine (AU)-rich elements in the untranslated regions (UTRs) of target mRNAs and destabilize the transcripts [7]. Such a capability enables TTP family members to play an important role in the maintenance of normal physiology by decreasing the expression of genes which cause pathological outcomes when excessively expressed. For example, while TTP can bind and destabilize tumor necrosis factor $\alpha(\mathrm{TNF} \alpha)$ mRNA to decrease the production of this pro-inflammatory cytokine [8], TTP- mice produce abundant TNF $\alpha$, and show symptoms of systemic inflammation [8]. Other than inflammation, TTP family members have also been found to regulate fetoplacental development and hematopoiesis, and may also act as tumor suppressors [9-11].

To search for potential regulators of the age-related bone loss, we have recently analyzed the gene expression profiles of human bmMSCs derived from donors of varying ages, and identified a list of potential ageassociated genes [12]. We found that ZFP36L1, but not $T T P$ and $Z F P 36 L 2$, was one of those genes. Interestingly, it was reported that ZFP36L1 expression in osteoblasts was involved in the parathyroid hormone-dependent bone remodeling [13]. These findings suggested a putative role of ZFP36L1 in the maintenance of bone homeostasis and in the etiology of age-related bone loss. However, the role of ZFP36L1 in the regulation of bone formation has not been addressed. ZFP36L1 knockout model was not able to answer this question because it resulted in embryonic lethality. In this regard, studies on the subjects, such as ZFP36L1 expression in bones in relation to age, the impact of ZFP36L1 to osteoblastic and adipogenic differentiation, are expected to provide clues to answer the question. Herein, we investigated the regulatory role of ZFP36L1 in the differentiation of MC3T3-E1 preosteoblasts, 3T3L1 preadipocytes, and multipotent $\mathrm{C} 3 \mathrm{H} 10 \mathrm{~T} 1 / 2$ cells. Our data indicated that ZFP36L1 could act as an enhancer of osteoblastic differentiation but a repressor of adipogenic differentiation, supporting the notion that decreased ZFP36L1 expression in bone marrow stem cells might contribute to aging-related bone loss.

\section{RESULTS}

\section{ZFP36L1 expression was down-regulated in the femurs and bone marrow mesenchymal stem cells (bmMSCs) of aged rats}

To examine if ZFP36L1 expression in bones changed with aging, we examined the expression of Zfp36l1 mRNA in femurs of adult (6-month-old) and aged (18 22-month-old) rats. Real-time quantitative PCR (RT-qPCR) analyses showed that Zfp36l1 mRNA level in femurs of aged rats $(n=6)$ was approximately $54.8 \%$ of those of adult rats $(n=3)$ (Figure 1A). Next, we pooled bmMSCs isolated from 6-month-old $(n=7)$, 20-monthold $(n=6)$, and 22-month-old $(n=4)$ rats into adult-1, aged-1, and aged-2 groups, respectively, and examined their Zfp36l1 expression. RT-qPCR analyses showed that Zfp36l1 mRNA levels in aged-1 and aged-2 groups were approximately $48 \%$ and $67 \%$, respectively, of those in adult-1 group (Figure 1B).

\section{ZFP36L1 enhanced osteoblastic differentiation of MC3T3-E1 preosteoblasts and multipotent C3H10T1/2 cells}

Next, to access the role of ZFP36L1 in osteoblastogenesis, we examined the effect of Zfp36ll knockdown on the differentiation of MC3T3-E1 preosteoblasts. We established Zfp36l1-knockdown (sh36L1) and control (shEV) cells. Zfp36l1 mRNA expression of sh36L1 cells was approximately 50\% less than that of shEV cells (Figure 2A). We induced cells to undergo osteoblastic differentiation and harvested cells at varying time periods for the measurement of osteocalcin and osteopontin expression. RT-qPCR analyses showed that while osteogenic induction induced dramatic increase of osteocalcin and osteopontin mRNAs around days 3 7 post-induction, such induction was repressed by Zfp36l1 knockdown (Figure 2B). Further, we established ZFP36L1-ovexpressing (ZFP36L1) and control (EV) MC3T3-E1 cells. Western blot analyses showed that the level of ZFP36L1 expression in transfected cells was approximately 1.5 fold of that of control cells (Figure 2C). We induced cells to undergo osteoblastic differentiation and examined the calcium precipitation in these cells 4 and 14 days post-induction. As evidenced by the results of Alizarin Red S staining, ZFP36L1-overexpressing cells exhibited stronger differentiation activity than EV cells on day 14 post induction (Figure 2D).

Next, to examine if ZFP36L1 also regulated osteoblastic differentiation of $\mathrm{C} 3 \mathrm{H} 10 \mathrm{~T} 1 / 2$ cells, we overexpressed ZFP36L1 in C3H10T1/2 cells, and selected two clones (clones 2 and 21) that expressed high levels of Zfp36l1 mRNA. Western blot analyses 


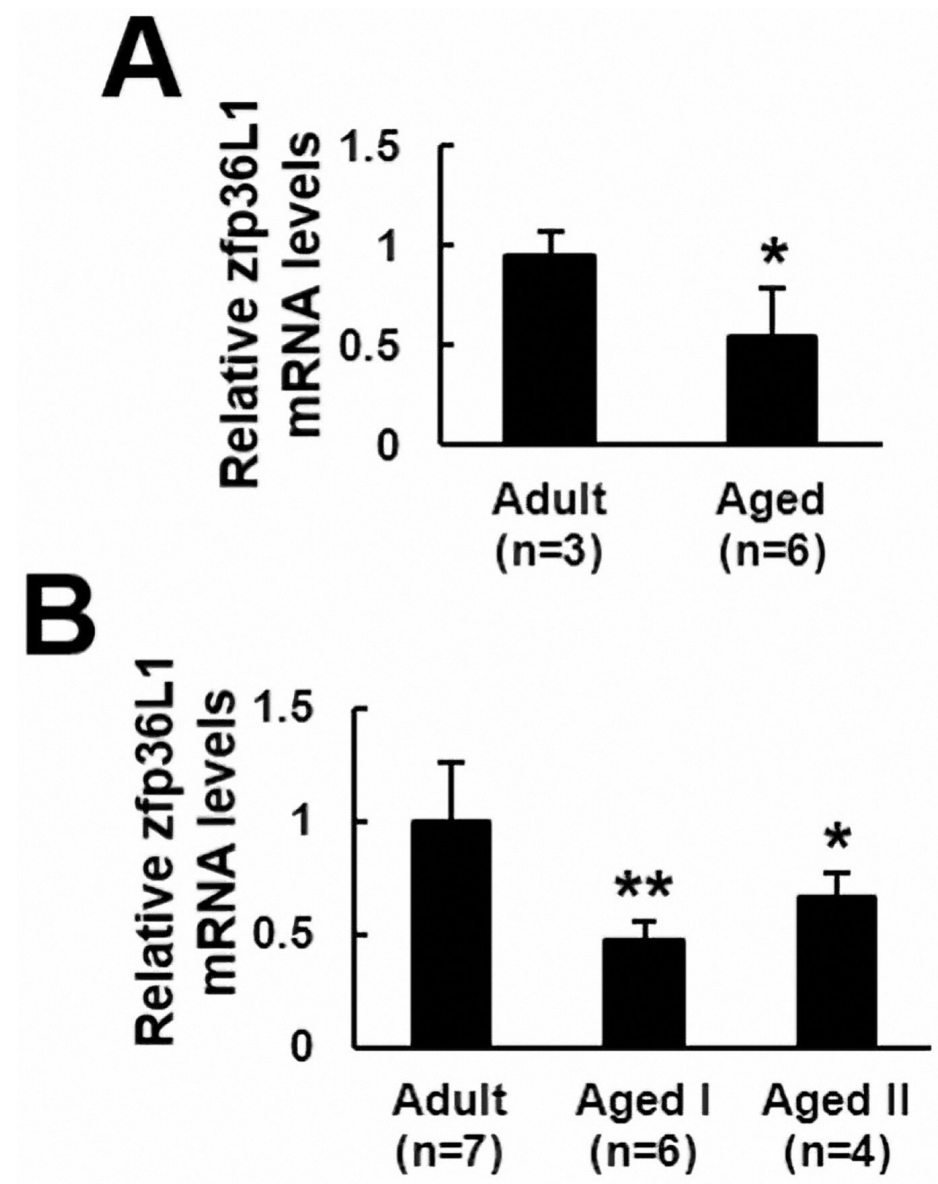

Figure 1: ZFP3611 expression in the femurs and bmMSCs of adult and aged rats. Total RNAs were isolated from the femurs A. and bone marrow mesenchymal stem cells B. of adult and aged rats, and were subjected to reverse transcription and RT-qPCR analyses for the expression of Zfp36l1 mRNA. *, $P<0.05$; **, $P<0.001$ versus adult control.
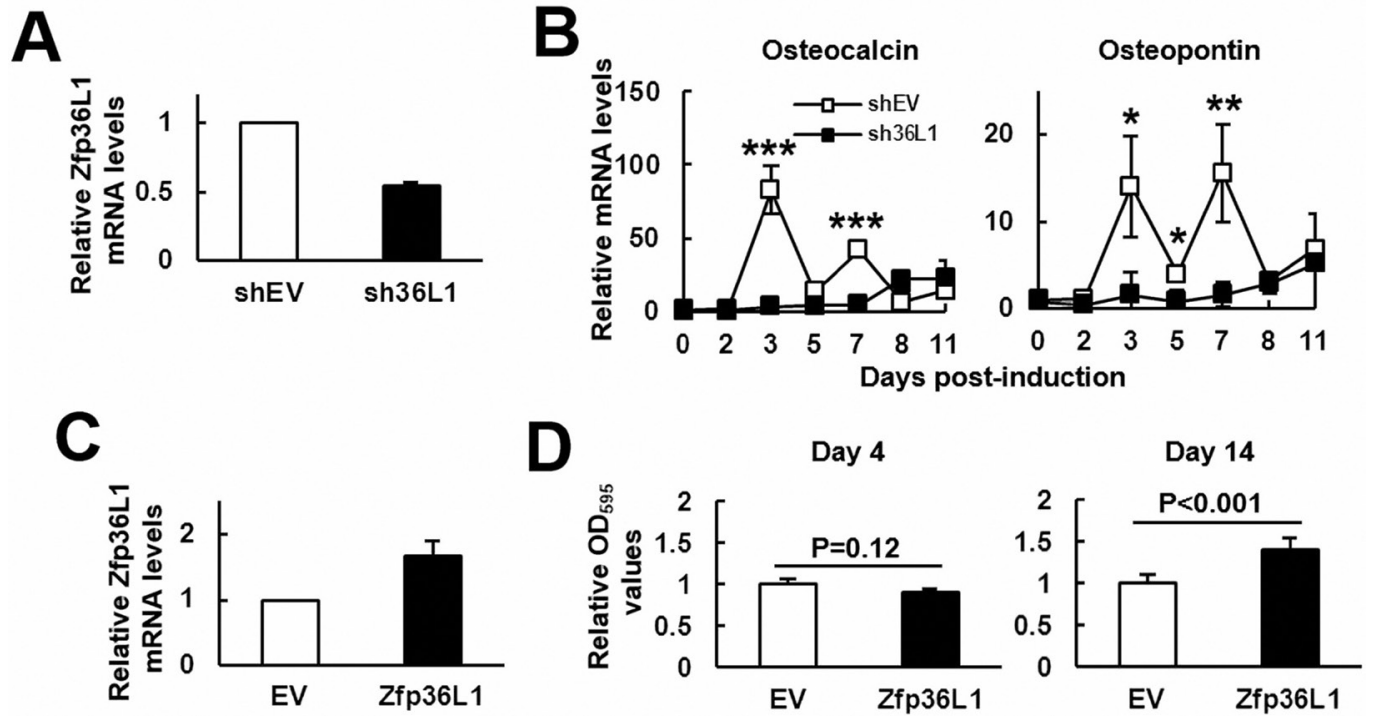

Figure 2: ZFP36L1 regulated osteoblastic differentiation of MC3T3-E1 preosteoblasts. A. RT-qPCR analyses showed the ZFP36L1 expression of ZFP36L1-knockdown (sh36L1) cells in relation to that of control (shEV) cells. B. RT-qPCR analyses. Confluent shEV and sh36L1 cells were induced to undergo osteoblastic differentiation, and the kinetic expression of osteocalcin and osteopontin mRNAs were shown. *, $P<0.05$; **, $P<0.01$; ***, $P<0.001$ versus shEV control at day 0 . C. RT-qPCR analyses showed the ZFP36L1 expression of ZFP36L1-overexpressing (ZFP36L1) cells in relation to that of control (EV) cells. D. Quantification of Alizarin Red S stains. Confluent EV and ZFP36L1 cells were induced to undergo osteoblastic differentiation. Cells were stained with Alizarin Red S on days 4 and 14 post-induction. The stains were dissolved and quantitated. 
showed that ZFP36L1 levels in clones 2 and 21 were approximately 2 and 2.5 fold of those in control cells (Figure 3A). We induced cells to undergo osteoblastic differentiation for 21 days. Alizarin Red S staining suggested that the differentiation activity in clones 2 and 21 was approximately 1.6 and 2 fold, respectively, of that of control cells (Figure 3B). In parallel, we examined the kinetic expression of osteocalcin, osteopontin, and Runx 2 mRNAs in clone 21 and control cells by RT-qPCR analyses. In general, data showed that clone 21 expressed more of those mRNAs than control cells (Figure 3C). Moreover, we seeded clone 21 and control cells into

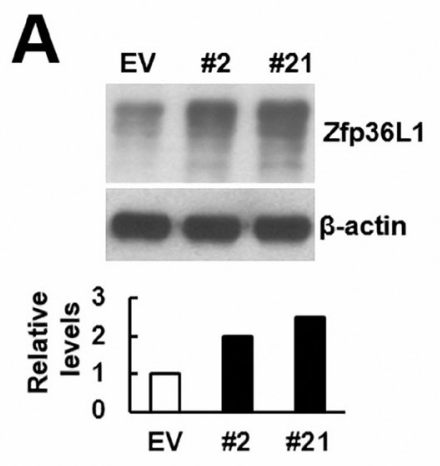

B

EV

\#2

\#21
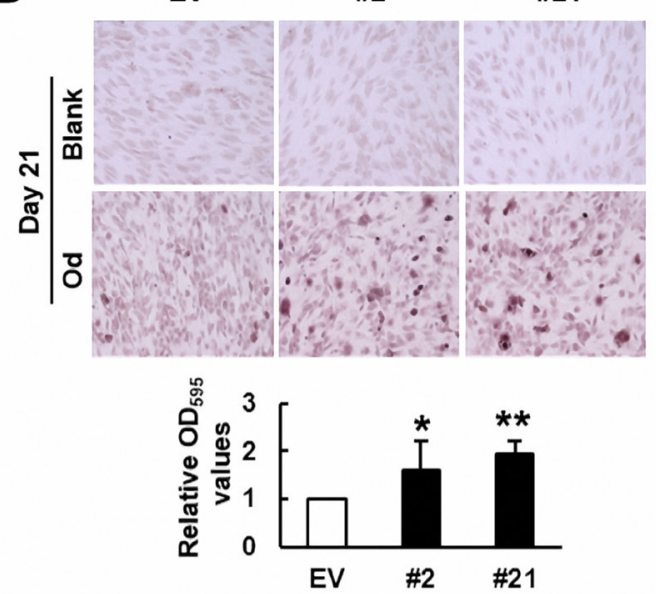

C

Osteopontin

Osteocalcin

高

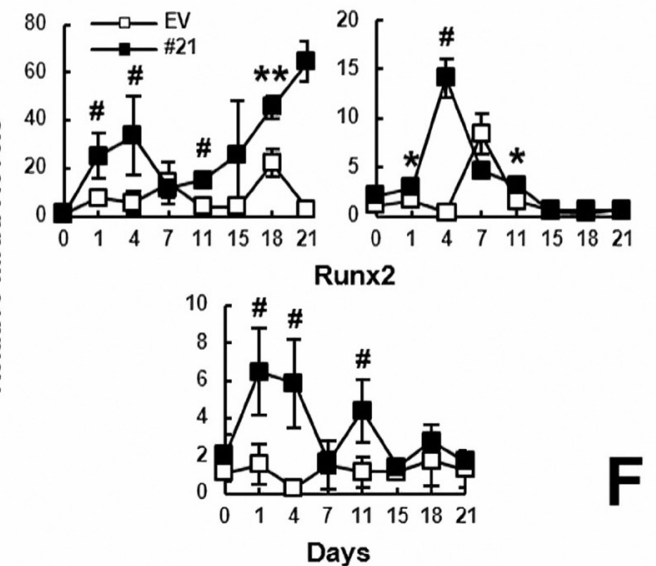

D

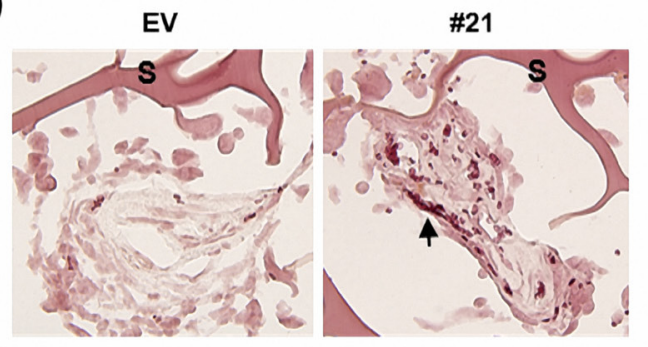

$\mathbf{E}$
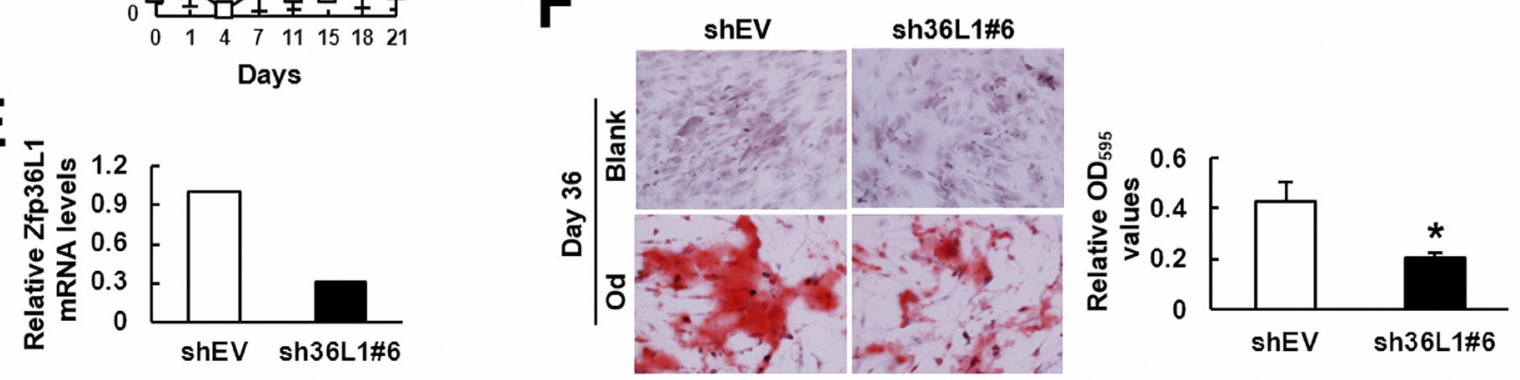

Figure 3: ZFP36L1 regulated osteoblastic differentiation of multipotent C3H10T1/2 cells. A. Western blot analyses. ZFP36L1 protein levels of EV control, clone 2 (\#2), and clone 21 (\#21) cells were detected and quantitated. Relative levels were calculated by comparing the signals of \#2 and \#21 to that of EV cells (to which a value of 1 was assigned). B. Osteoblastic induction. EV, \#2, and \#21 cells were either left untreated (blank) or induced to undergo osteoblastic differentiation (od). Cells were stained with Alizarin Red S 21 days post-induction. Representative photos are shown. The stains were quantitated, and the signals of \#2 and \#21 were compared to that of EV cells (to which a value of 1 was assigned). ${ }^{*}, P<0.05$; ${ }^{*}, P<0.005$ versus EV control. C. RT-qPCR analyses. The expression kinetics of osteocalcin, osteopontin, and Runx 2 mRNAs in cells after osteoblastic induction were examined, and presented in relative to EV control of day 0 (to which a value of 1 was assigned). ${ }^{*}, P<0.05 ;{ }^{* *}, P<0.005 ;{ }^{*}, P<0.001$ versus EV control. D. Ex vivo experiments and histological analyses. EV and \#21 cells were seeded into 3 scaffolds ( 4 x $10^{5}$ cells/scaffold) separately, and implanted in pairs into the back of 3 nude mice. Implants were retrieved 4 weeks after implantation and prepared for histological analysis. Histological sections were stained with Alizarin Red S. Representative images are shown. Scaffold was indicated by S. Stained cells were indicated by arrowhead. E. RT-qPCR analyses showed the ZFP36L1 expression of ZFP36L1-knockdown clone 6 (sh36L1\#6) cells in relation to that of control (shEV) cells. F. Osteoblastic induction. shEV and sh36L1\#6 cells were either left untreated (blank) or induced to undergo osteoblastic differentiation (od). Cells were stained with Alizarin Red S 36 days post-induction. Representative photos are shown. The stains were solubilized and quantitated. * $P<0.05$ versus shEV control. 
scaffolds separately, and implanted subcutaneously into nude mice for 4 weeks. We stained the histological sections of retrieved implants with Alizarin Red S. Unfortunately, cells grew so crowdedly in implants that we were not able to count DAPI-stained cells. However, we observed that ZFP36L1 overexpression seemed to increase the numbers of Alizarin Red S-stained cells (Figure 3D). These results suggested that ZFP36L1 overexpression promoted osteoblastic differentiation of C3H10T1/2 cells. Next, we prepared ZFP36L1-knockdown clone 6 cells whose Zfp $36 l 1$ mRNA levels were approximately 30\% of that of the corresponding control cells (Figure 3E). We induced cells to undergo osteoblastic differentiation, and stained cells with Alizarin Red S 36 days post-induction. The results showed that ZFP36L1-knockdown decreased osteoblastic differentiation activity (Figure 3F). Taken together, our data indicated ZFP36L1 as an enhancer of osteoblastic differentiation.

\section{ZFP36L1 repressed adipogenic differentiation of 3T3-L1 preadipocytes and $\mathrm{C} 3 \mathrm{H} 10 \mathrm{~T} 1 / 2$ cells}

We induced C3H10T1/2 and 3T3-L1 cells to undergo adipogenic differentiation, and examined the kinetic expression of Zfp36l1 mRNA levels. The results showed that the expression of Zfp $36 l 1$ mRNA decreased with the advance of differentiation in these cells (Supplemental Figure S1). To assess the role of ZFP36L1 in adipogenic differentiation, we established ZFP36L1-ovexpressing and control 3T3-L1 cells. Western blot analyses showed that the ZFP36L1 level of ZFP36L1-ovexpressing cells was approximately 2.2 fold of that of control cells (Figure 4A). We induced cells to undergo adipogenic differentiation for various days, and examined lipid droplet formation as well as Ppary 2 mRNA expression. As evidenced by Oil Red $\mathrm{O}$ staining performed 5 days post induction, ZFP36L1-ovexpressing cells showed approximately 28\% less lipid droplet formation than control cells (Figure 4B). RT-qPCR analyses showed that adipogenic induction increased Ppary 2 expression in both groups of cells; however, ZFP36L1 overexpression significantly repressed Ppary 2 expression 4 days post induction (Figure 4C). We also prepared ZFP36L1-knockdown cells whose Zfp36l1 mRNA levels were approximately $72 \%$ less than control 3T3-L1 cells (Figure 4D), and induced cells to undergo adipogenic differentiation for various days. RT-qPCR analyses showed that ZFP36L1 knockdown potentiated the mRNA expression of PPAR $\gamma 2$, aP2, and adiponectin (Figure 4E).

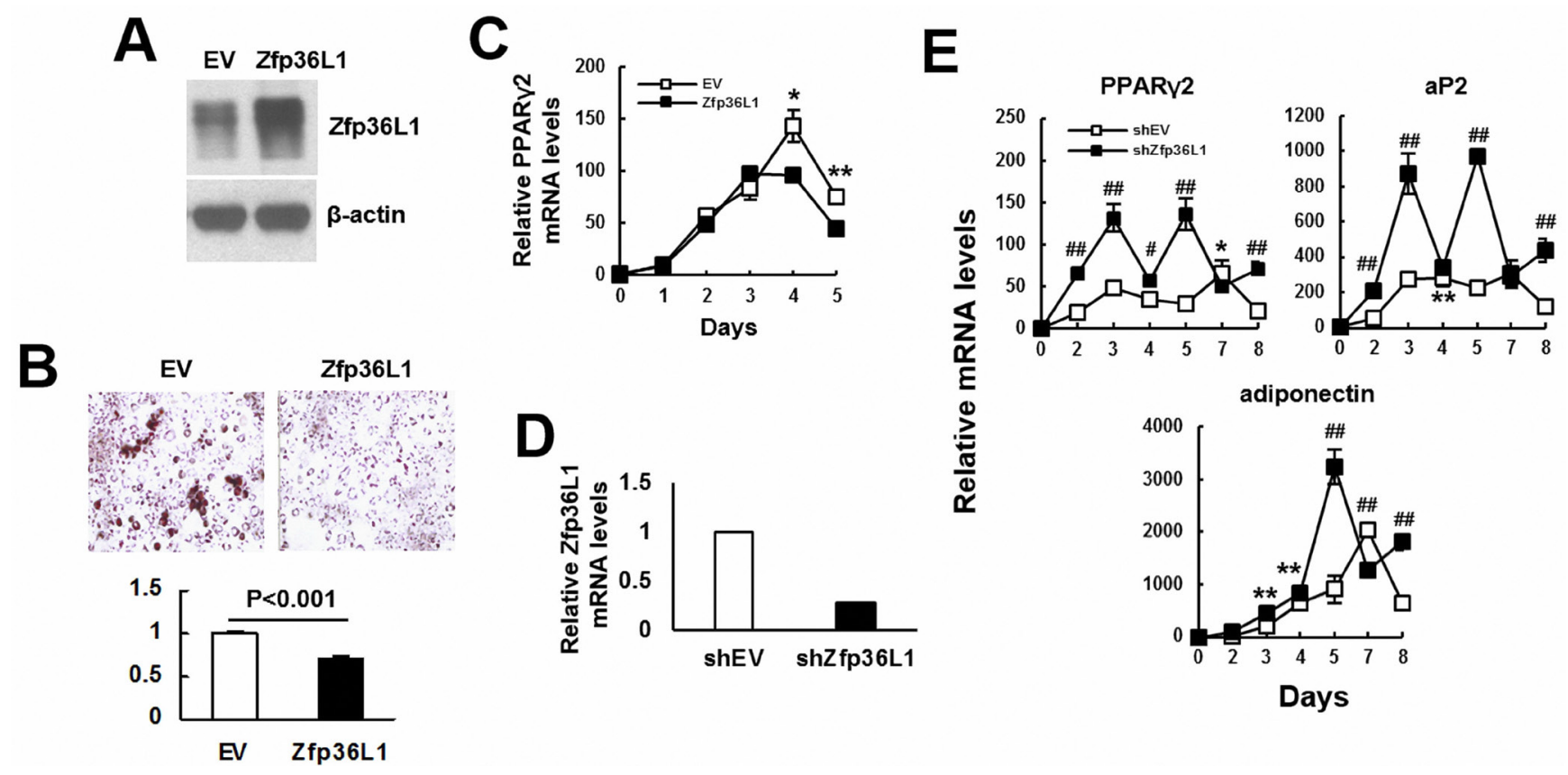

Figure 4: ZFP36L1 regulated adipogenic differentiation of 3T3-L1 preadipocytes. A. Western blot analyses. ZFP36L1 protein levels of EV control and ZFP36L1-overexpressing (ZFP36L1) 3T3-L1 cells were shown. B. Adipogenic induction. EV and ZFP36L1 cells were induced to undergo adipogenic differentiation. Cells were stained with Oil Red O 5 days post-induction. Representative photos are shown. The stains were quantitated, and the signal of ZFP36L1 cell was compared to that of EV cell (to which a value of 1 was assigned). C. RT-qPCR analyses. The expression kinetics of Ppary 2 mRNA in cells after adipogenic induction were examined, and presented in relative to EV control of day 0 (to which a value of 1 was assigned). ${ }^{*}, P<0.05$; **, $P<0.01$ versus EV control. D. RT-qPCR analyses. ZFP36L1 expression of ZFP36L1-knockdown (shZFP36L1) cells in relation to that of control (shEV) cells was shown. E. RT-qPCR analyses. shEV and shZFP36L1 cells were induced to undergo adipogenic differentiation. The expression kinetics of Ppar 2 , aP2, and adiponectin mRNAs in cells after adipogenic induction were examined, and presented in relative to EV control of day 0 (to which a value of 1 was assigned). *, $P<0.05 ; * *, P<0.01$;,$P<0.001 ;{ }^{\#}, P<0.0001$ versus $\mathrm{EV}$ control. 
Next, we examined the impact of ZFP36L1 overexpression to the adipogenic differentiation of the ZFP36L1-overexpressing C3H10T1/2 clones 2 and 21. We induced cells to undergo adipogenic differentiation for various days. Oil Red O staining performed 8 days post-induction showed that the accumulated lipid droplets in clones 2 and 21 was approximately $67 \%$ and $36 \%$, respectively, of that of control cells (Figure 5A). In parallel, RT-qPCR analyses showed that adipogenic induction increased the mRNA levels of aP2 and adiponectin, whereas ZFP36L1 overexpression repressed expression of these genes in a dose-dependent manner (Figure 5B). Moreover, comparison of the kinetic expression of Ppary 2 mRNA in clone 21 and control cells also showed decreased PPAR $\gamma 2$ expression in clone 21 cells (Figure 5C). Subsequently, we seeded clone 21 and control cells into scaffolds separately, and implanted subcutaneously into nude mice for 2 weeks. We stained the histological sections of retrieved implants with DAPI and Oil Red O, and to estimate the percentage of Oil Red O-stained cells in the histological sections. Our data showed that approximately $51 \%$ and $27 \%$ of DAPI-

A

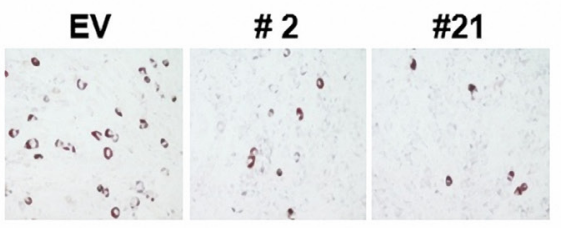

B
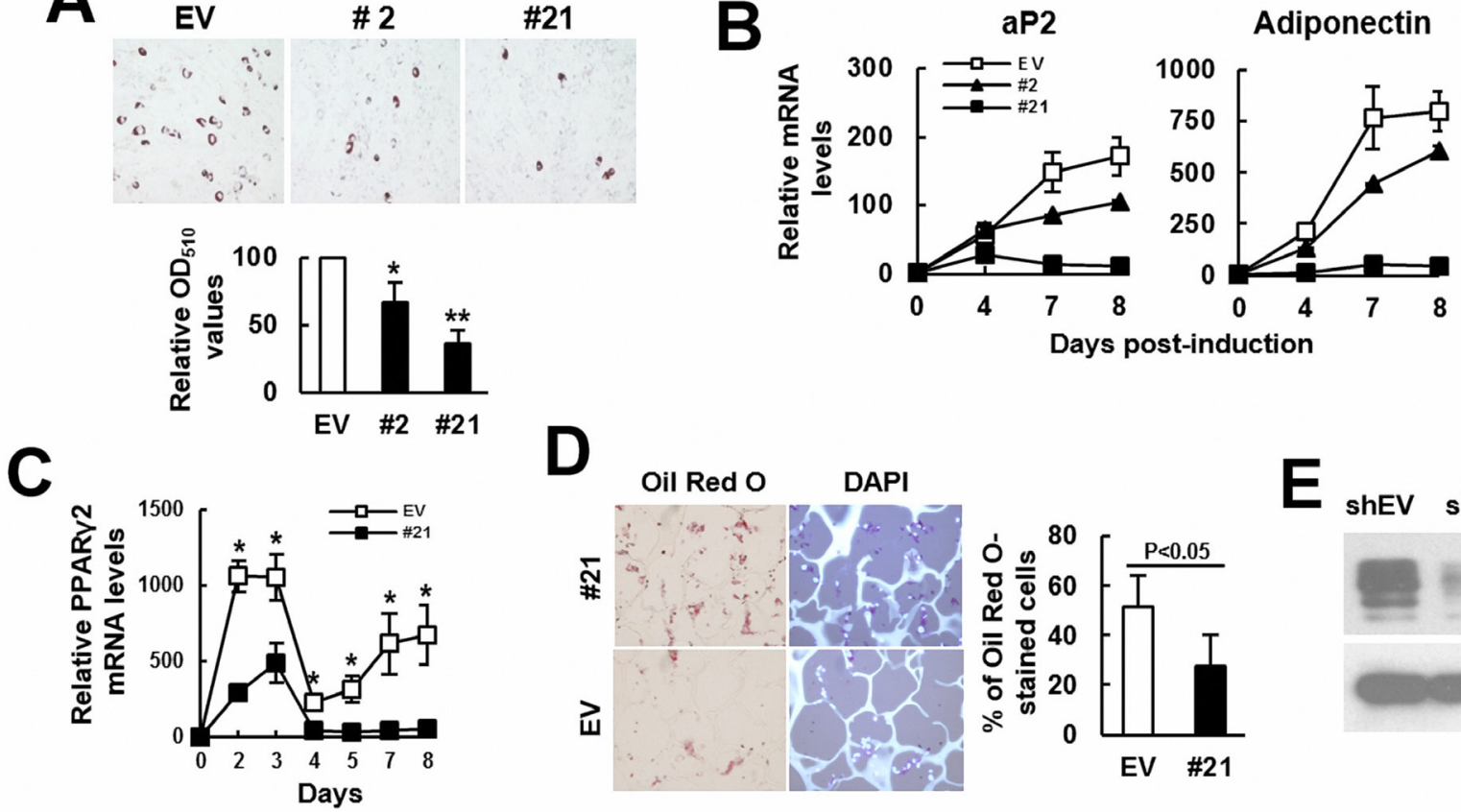

D
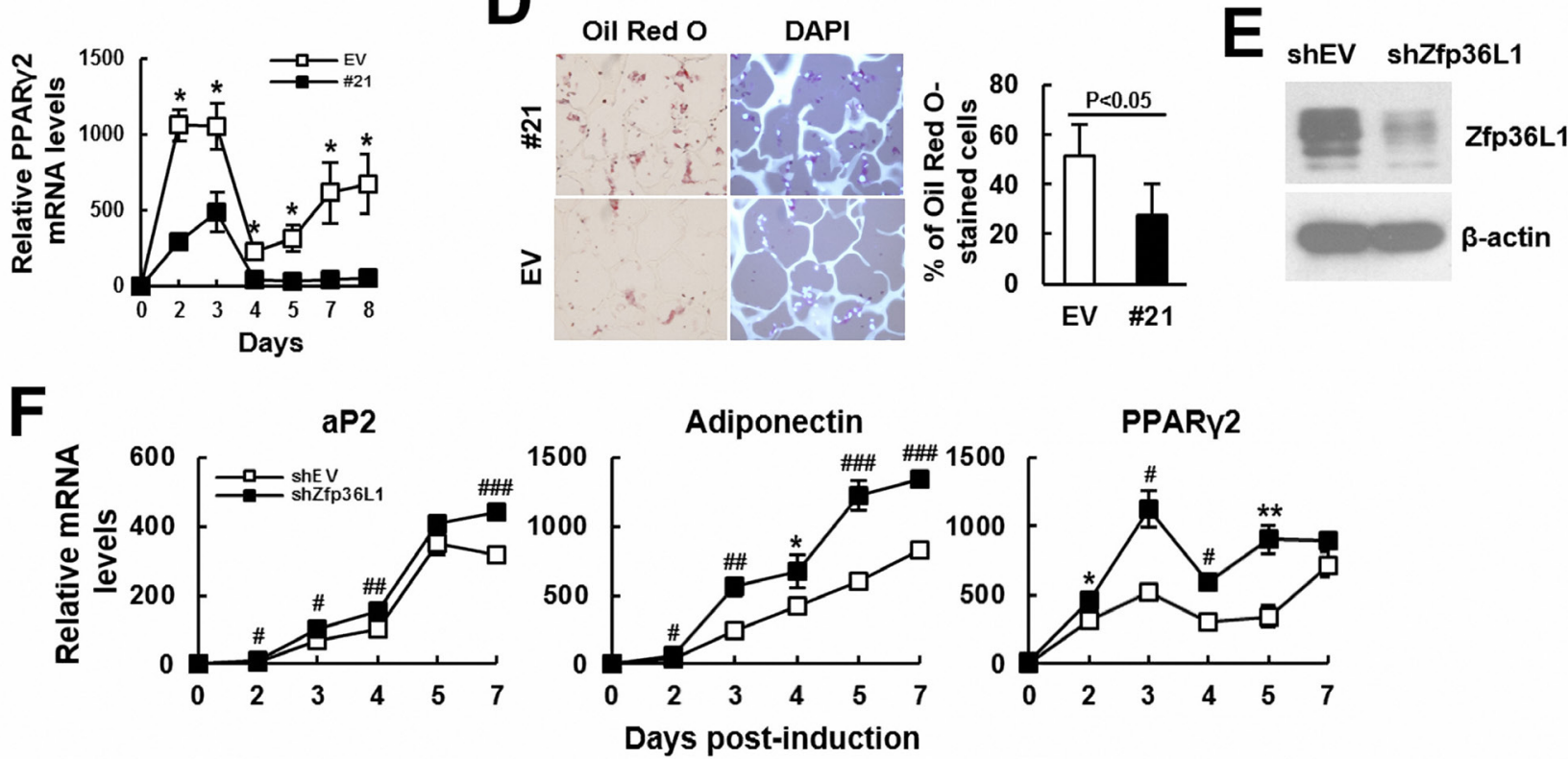

Figure 5: ZFP36L1 regulated adipogenic differentiation of C3H10T1/2 cells. A. Adipogenic differentiation. C3H10T1/2 control (EV), clone 2 (\#2), and clone 21(\#21) cells were induced to undergo adipogenic differentiation. Cells were stained with Oil Red O 8 days post-induction. Representative photos are shown. The stains were quantitated, and the signals of \#2 and \#21 were compared to that of EV cells (to which a value of 100 was assigned). ${ }^{*}, P<0.05 ; * *, P<0.005$ versus EV control. B. RT-qPCR analyses. The expression kinetics of $a P 2$ and adiponectin mRNAs in cells after adipogenic induction were examined, and presented in relative to EV control of day 0 (to which a value of 1 was assigned). C. RT-qPCR analyses. The expression kinetics of Ppar 2 mRNA in EV and \#21 cells after adipogenic induction were shown. *, $P<0.05$ versus $\mathrm{EV}$ control. D. Ex vivo experiments and histological analyses. EV and \#21 cells were seeded into 3 scaffolds ( $4 \times 10^{5}$ cells/scaffold) separately, and implanted in pairs into the back of 3 nude mice. Implants were retrieved 2 weeks after implantation and prepared for histological analysis. Histological sections were stained with Oil Red O and DAPI. Representative images are shown. The ratio of Oil Red O-stained cells were calculated. E. Western blot analyses. ZFP36L1 expression of ZFP36L1-knockdown (shZFP36L1) and control (shEV) C3H10T1/2 cells were shown. F. RT-qPCR analyses. shEV and shZFP36L1 cells were induced to undergo adipogenic differentiation. The expression kinetics of $a P 2$, adiponectin, and $P$ par 2 mRNAs were shown. ${ }^{*}, P<0.05 ; * * . P<0.01$;,$P<$ 0.005 ; \#, $P<0.001 ;$;,$P<0.0001$ versus corresponding EV control. 
stained cells in control and clone 21 groups, respectively, accumulated lipid droplets (Figure 5D), indicating that ZFP36L1 overexpression inhibited adipogenic differentiation of C3H10T1/2 cells. On the other hand, we prepared ZFP36L1-knockdown cells whose ZFP36L1 levels were approximately $72 \%$ less than control cells (Figure 5E), and induced cells to undergo adipogenic differentiation for various days. RT-qPCR analyses showed that Zfp36L1 knockdown increased the mRNA levels of aP2, adiponectin and PPAR $\gamma 2$ (Figure 5F).

Next, we examined if troglitazone (TZD), a PPAR $\gamma 2$ agonist, prevented ZFP36L1 from inhibiting adipogenic differentiation. Our data showed that while ZFP36L1 overexpression inhibited lipid droplets formation and the expression of PPAR $\gamma 2$, aP2, and adiponectin, TZD treatment restored the adipogenic differentiation (Figure 6). These evidences suggested the significance of PPAR $\gamma 2$ repression in ZFP36L1's inhibitory effect on adipogenic differentiation.

\section{ZFP36L1 regulated PPAR $\gamma 2$ expression by post- transcriptional mechanism(s)}

To elucidate how ZFP36L1 regulated lineage differentiation, we focused on how ZFP36L1 regulated PPAR $\gamma 2$ expression. We overexpressed ZFP36L1 followed by an empty luciferase reporter or a reporter driven by Ppary promoter in parental cells transiently, and induced cells to undergo adipogenic differentiation, and harvested cells 0,1 , and 2 days post-induction. RTqPCR analyses showed that the induction of PPAR $\gamma 2$ expression as shown in the control cells was repressed in cells receiving ZFP36L1 (Figure 7A). Parallel luciferase assays showed that the Ppary promoter activity in cells receiving $\mathrm{ZFP} 36 \mathrm{~L} 1$ was no less than that of control cells, indicating that ZFP36L1 did not inhibit Ppary promoter activity (Figure 7B). Taken together, these data indicated that ZFP36L1 might inhibit PPAR 22 expression at the post-transcriptional level. So, we performed biotin pulldown assays using unlabeled and biotin-labeled 3'UTR of Ppary 2 mRNA to examine the interaction between ZFP36L1 and Ppary2 mRNA. Western blot analyses on the complexes pulled down by streptavidin-coated beads showed that ZFP36L1 was detected in the complexes reacting with biotin-labeled transcript but not in those reacting with unlabeled transcript, which indicated the binding specificity of the streptavidin-coated beads, and suggested the binding of endogenous ZFP36L1 to Ppary 2 mRNA (Figure 8A). The 3'UTR of Ppary2 mRNA contains several AU-rich fragments (Figure 8B). To further address the binding between ZFP36L1 and Ppar 2 mRNA, we performed electrophoretic mobilityshift assays. We prepared the cytoplasmic components of the cell lysates prepared from the control and the cells transiently overexpressed ZFP36L1 (Figure 8C) and incubated these components with biotin-labeled 3'UTR of Ppary 2 mRNA. As shown in Figure 8D, cytoplasmic lysates of the control cells bound to the biotin-labeled RNA probes. A 30-fold molar excess of unlabeled RNA

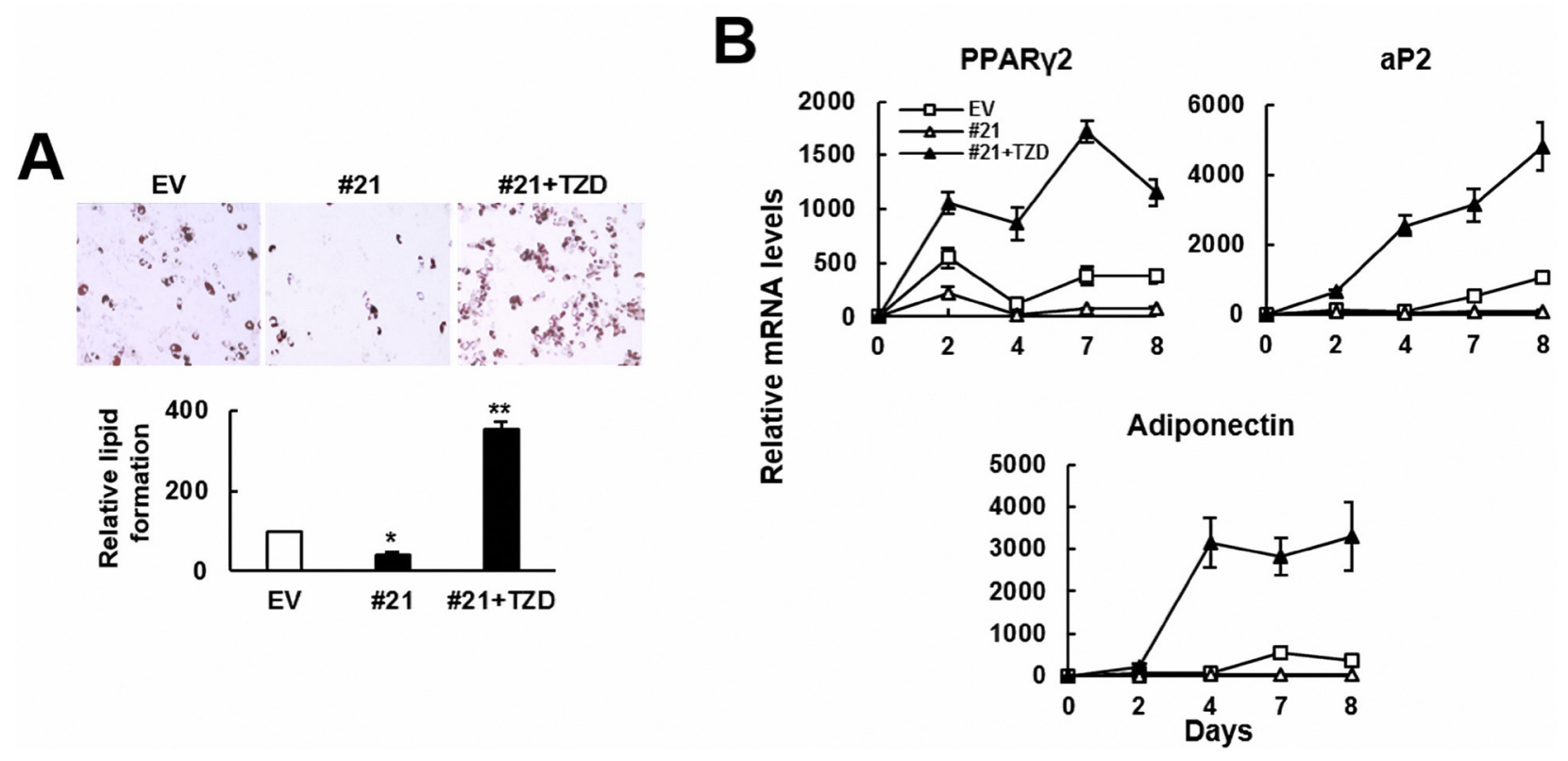

Figure 6: TZD prevented ZFP36L1 from inhibiting adipogenic differentiation of C3H10T1/2 cells. A. Adipogenic differentiation. Control (EV) and ZFP36L1-overexpressing C3H10T1/2 \#21 cells (\#21) were induced to undergo adipogenic differentiation, and \#21 cells were either left untreated or concomitantly treated with troglitazone (TZD, $1 \mu \mathrm{M})$. Cells were stained with Oil Red O 8 days post-induction. Representative photos are shown. The stains were quantitated, and the signals of \#21 cells were compared to that of EV cells (to which a value of 100 was assigned). ${ }^{*}, P<0.05$; **, $P<0.00005$ versus EV control. B. RT-qPCR analyses. The expression kinetics of Ppary2, $a P 2$, and adiponectin mRNAs in cells after adipogenic induction were shown. 


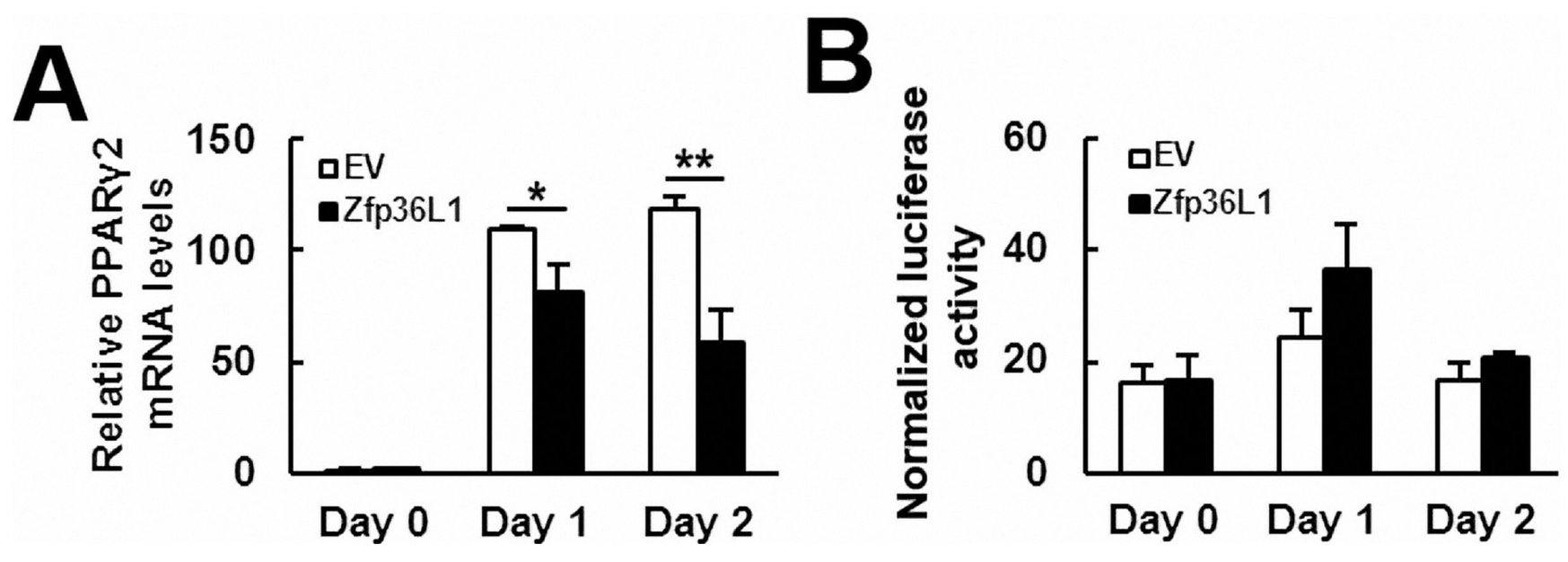

Figure 7: ZFP36L1 decreased PPAR 2 expression but not Ppary promoter activity. ZFP36L1-overexpressing (ZFP36L1) and control (EV) C3H10T1/2 cells were transfected with a luciferase reporter driven by Ppary promoter and a Renilla control reporter, cells were then induced to undergo adipogenic differentiation, and were harvested at the times indicated. A. RT-qPCR analyses. The expression of Ppary 2 mRNA was examined. Data represent the mean \pm S.D. from three experiments. ${ }^{*}, P<0.05 ;{ }^{* *}, P<0.005$ versus control. B. Luciferase assays. Cells harvested after adipogenic induction were analyzed for luciferase and Renilla activities. The normalized luciferase signals of EV and ZFP36L1 cells were shown.

A

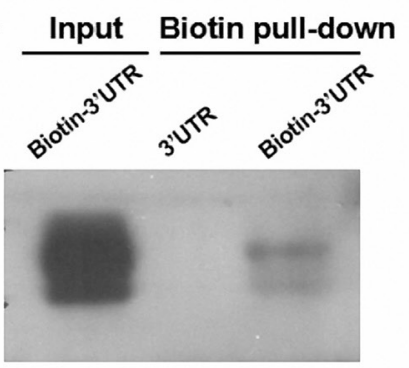

C

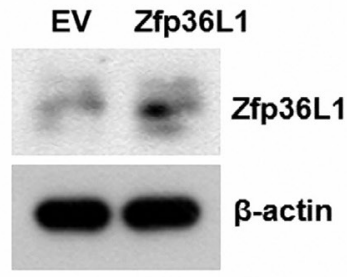

B

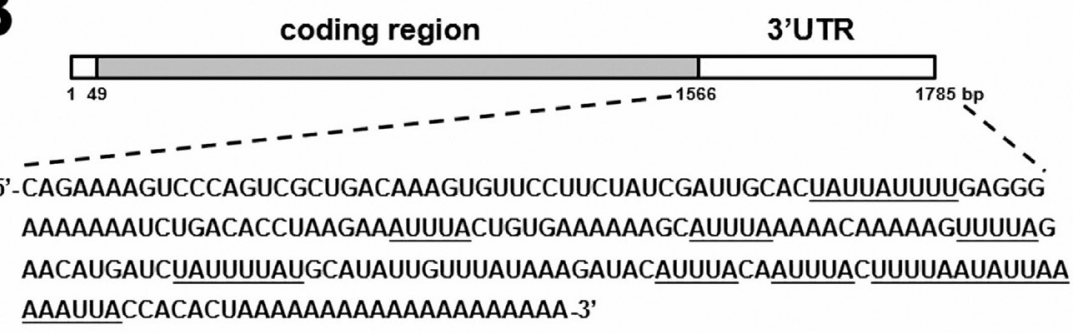

D

$\begin{array}{rrrcc}\text { Biotin-3'UTR } & + & + & + & + \\ \text { 3'UTR } & - & - & 30 \mathrm{x} & -\end{array}$

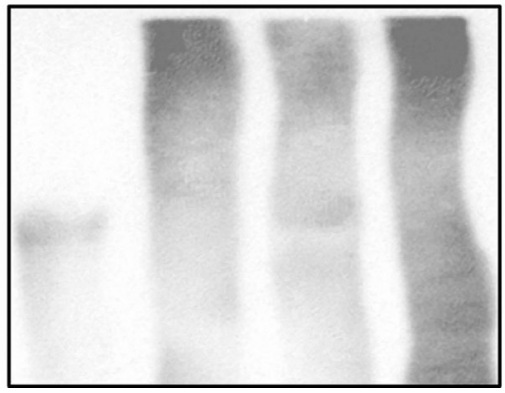

Figure 8: Interactions of ZFP36L1 and Ppary2 transcripts. A. Biotin pull-down assays. C3H10T1/2 cells were lysed, and $150 \mu \mathrm{g}$ of cell lysates were incubated with either biotin-labeled (biotin-3'UTR) or unlabeled (3'UTR) transcripts derived from the Ppar $\gamma 2$ mRNA, and were subjected to pull-down assays followed by Western blot analyses to detect ZFP36L1 expression. Forty micrograms of whole-cell lysate was used as input. B. Schematic representation of the full-length Ppar $22 \mathrm{mRNA}$. The sequence of the 3'UTR is shown, and in which the putative AU-rich fragments are underlined. C. Western blot analyses. The ZFP36L1 levels of EV control cells and cells transiently overexpressing ZFP36L1 (ZFP36L1) were examined. D. RNA EMSA assays. Ten-micrograms of cytoplasmic fractions prepared from control (EV) and ZFP36L1-overexpressing (ZFP36L1) cells were reacted with biotin-labeled 3'UTR of Ppary2 mRNA. A 30-molar excess of unlabeled 3'UTR probes was added for competition experiments. f, biotin-labeled transcripts without incubation with cell lysate. 
probes decreased the binding to the biotin-labeled RNA probes, which resulted in the appearance of unbound free biotin-labeled RNA probes. In addition, cytoplasmic lysates of the ZFP36L1-overexpressing cells exhibited stronger RNA-binding activity than the lysates of control cells (Figure 8D). Our data suggested the binding of ZFP36L1 to the 3'UTR of Ppary2 mRNA. Subsequently, we prepared Flag-tagged ZFP36L1-overexpressing (F-ZFP36L1) and control (F-EV) C3H10T1/2 cells (Figure 9A), and induced these cells to undergo adipogenic differentiation for $24 \mathrm{~h}$ at the presence of troglitazone. We harvested cells $24 \mathrm{~h}$ post-induction. RTqPCR assays revealed that troglitazone induced equal amounts of Ppary 2 mRNA in F-EV and F-ZFP36L1 cells (Figure 9B). Then, we performed immunoprecipitation experiments using anti-Flag or control antibody to pull down the ribonucleoprotein complexes in equal amounts of lysates prepared from these cells. RNAs were extracted from these immunoprecipitates, and subjected to RTqPCR analyses for Ppar 2 and $\beta$-actin mRNAs. The results showed that in F-EV cells, there was no significant difference in the amounts of Ppary 2 mRNA between the ribonucleoprotein complexes pulled down by anti-Flag and control antibody, respectively (Figure 9C). However, in F-ZFP36L1 cells, the levels of Ppary 2 mRNA in the ribonucleoprotein complexes pulled down by anti-Flag antibody was $40 \%$ less than that in the ribonucleoprotein complexes pulled down by control antibody (Figure 9C). A possible explanation for this result is that the F-ZFP36L1containing protein complexes were able to enhance the degradation of Ppar 2 mRNA. Accordingly, we set out to examine the function of the 3'UTR of Ppary 2 mRNA in regulating gene expression in the background of ZFP36L1 overexpression. We replaced the SV40 poly(A) fragment which located 3' to the luciferase cDNA in pGL3-promoter vector by the 3'UTR of Ppary 2 mRNA to generate pGL3-
UTR reporter (Figure 10A). We established ZFP36L1overexpressing $\mathrm{C} 3 \mathrm{H}-\mathrm{ZFP} 36 \mathrm{~L} 1$ and control $\mathrm{C} 3 \mathrm{H}-\mathrm{EV}$ cells (Figure 10B), transfected these cells with pGL3UTR or control luciferase reporter, and then quantitated the levels of luciferase transcripts by RT-qPCR analyses. As examined 4 and $6 \mathrm{~h}$ post transfection, and compared with the luciferase transcript levels of C3H-EV cells receiving control pGL3 reporter, the levels of luciferase transcript in $\mathrm{C} 3 \mathrm{H}-\mathrm{EV}$ cells receiving pGL3-UTR reporter were not significantly changed (Figure 10C). On the other hand, compared with the luciferase transcript levels of C3H-ZFP36L1 cells receiving control pGL3 reporter, the levels of luciferase transcript in C3H-ZFP36L1 cells receiving pGL3-UTR reporter were approximately $29 \%$ and $37 \%$ lower, as examined 4 and $6 \mathrm{~h}$ post transfection, respectively (Figure 10C). We also induced $\mathrm{C} 3 \mathrm{H}-\mathrm{EV}$ and C3H-ZFP36L1 cells to undergo adipogenic differentiation at the presence of troglitazone which was removed 24 $\mathrm{h}$ post induction. We treated cells with Actinomycin D for $0,2,4,6$, and $8 \mathrm{~h}$, and examined the Ppary 2 mRNA levels. As shown in Figure 10D, Ppary2 mRNA levels in $\mathrm{C} 3 \mathrm{H}-\mathrm{EV}$ cells remained intact $2 \mathrm{~h}$ after Aitinomycin $\mathrm{D}$ treatment, and decreased $\sim 67 \%, \sim 85 \%$ and $\sim 76 \%$ at 4,6 , and $8 \mathrm{~h}$ after treatment, respectively. On the other hand, Ppary 2 mRNA levels in C3H-ZFP36L1 cells decreased $\sim 56 \%, \sim 86 \%, \sim 87 \%$, and $\sim 92 \%$ at $2,4,6$, and $8 \mathrm{~h}$ after Actinomycin D treatment. Taken together, our data suggested that ZFP36L1 might down-regulate the expression of Ppary2 mRNA by binding to the 3'UTR of this mRNA.

\section{DISCUSSION}

As ZFP36L1 is a RNA-binding protein, it is expected to be able to modulate the levels of its target
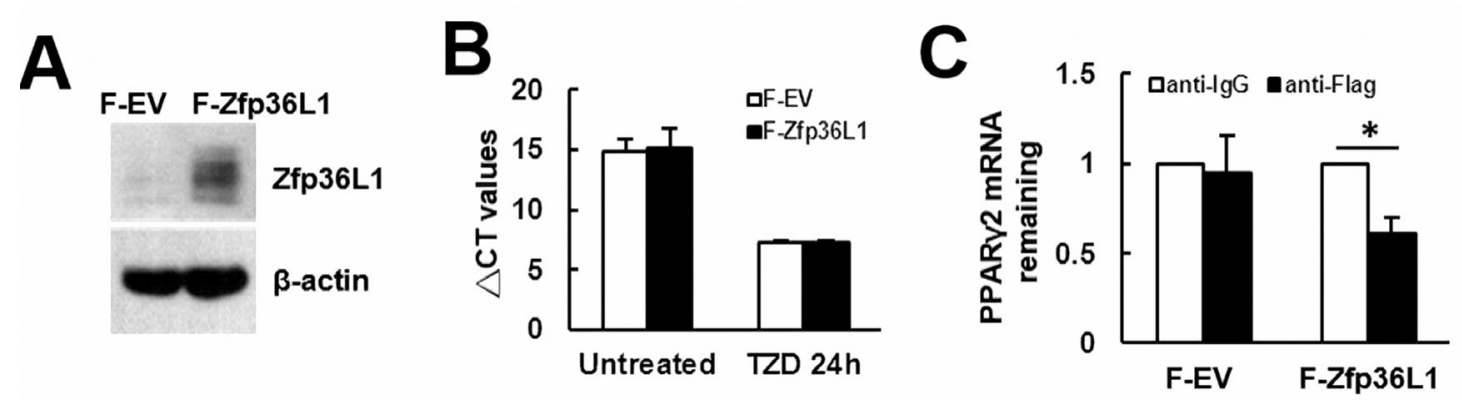

Figure 9: Examination of the binding of ZFP36L1-containing protein complexes on Ppary2 mRNA. A. Western blot analyses. The ZFP36L1 levels of C3H10T1/2 cells overexpressing Flag-tagged ZFP36L1 (F-ZFP36L1) and control cells (F-EV) were shown. B. RT-qPCR analyses. F-EV and F-ZFP36L1 cells were induced to undergo adipogenic differentiation with or without concomitant treatment of troglitazone (TZD, $1 \mu \mathrm{M}$ ) for $24 \mathrm{~h}$. The expression of Ppar $2 \mathrm{mRNA}$ was examined. C. Ribonucleoprotein immunoprecipitation and RT-qPCR analyses. One milligram of lysates prepared from F-EV or F-ZFP36L1 cells $\left(1 \mathrm{x} 10^{7}\right)$ were incubated with protein A beads precoated with $15 \mu \mathrm{g}$ of either anti-Flag or anti-IgG antibody to precipitate ribonucleoprotein complexes and to extract RNAs from the complexes as described in Materials and Methods. RNAs were used in subsequent RT-qPCR assays for Ppary 2 and $\beta$-actin mRNAs. Ppar 2 signals were normalized to $\beta$-actin signals. The normalized Ppar 2 signals obtained from the ribonucleoprotein complexes pulled down by anti-Flag antibody were compared with those pulled down by anti-IgG antibody (to which a value of 1 was assigned). Data represent the mean \pm S.D. from three analyses. ${ }^{*}, P<0.05$. 
transcripts and therefore participate in a wide spectrum of physiological activities. While ZFP36L1 and the other TTP members have been found to harbor antiinflammatory and anti-cancer capability, we have explored the potential involvement of ZFP36L1 in the regulation of aging-related bone loss.

It is well documented that aging bones contain less bone mass and osteoblasts than adult bones, which can be attributed to decreased bone formation in aging bones. Bone formation is carried out by osteoblasts which are differentiated from osteoprogenitors and bmMSCs. So, if ZFP36L1 played a role in the aging-related bone loss, it would be reasonable to assume that ZFP36L1 might express differentially in adult and aged bmMSCs, and that ZFP36L1 might regulate osteoblastic and adipogenic differentiation. Notably, our data showed that expression of Zfp36l1 mRNA was down-regulated in the femurs and bmMSCs of aged rats compared with those noted in adult rats (Figure 1). Moreover, by examining the impact of ZFP36L1 knockdown and overexpression on osteoblastic differentiation of MC3T3-E1 and C3H10T1/2 cells in vitro; and on the osteoblastogenic potential of C3H10T1/2 cells ex vivo (Figures 2 and 3), we showed that ZFP36L1 could act as an enhancer of osteoblastic differentiation. On the other hand, examinations of ZFP36L1 knockdown and overexpression on adipogenic differentiation of 3T3-
$\mathrm{L} 1$ and $\mathrm{C} 3 \mathrm{H} 10 \mathrm{~T} 1 / 2$ cells in vitro; and on the adipogenic potential of C3H10T1/2 cells ex vivo (Figures 4 and 5), we showed that ZFP36L1 could act as a repressor of adipogenic differentiation. Taken together, our findings support the notion that ZFP36L1 might participate in the maintenance of bone homeostasis, and that downregulation of ZFP36L1 expression in bmMSCs might be a part of the mechanisms underlying the aging-related bone loss.

Our studies also shed light on the mechanisms by which ZFP36L1 regulates lineage differentiation. Runx2 is the master transcriptional regulator of osteoblastic differentiation; it can promote osteoblastic differentiation by recruiting co-activators [14]. Our data showed that ZFP36L1 overexpression increased Runx2 mRNA expression (Figures 3C). Given that ZFP36L1 is a RNAbinding protein, and that binding of ZFP36L1 enhances the degradation of its target transcripts, ZFP36L1 is unlikely to increase Runx2 expression by stabilizing Runx 2 mRNA. How ZFP36L1 induces Runx2 expression, and more importantly, how critical the Runx2 induction is in the ZFP36L1-induced commitment to osteoblastic lineage are currently unclear. In comparison, however, it has been shown that PPAR $\gamma 2$ is a more dominant regulator of osteoblastic differentiation. PPAR $\gamma 2$ overexpression repress Runx2 expression and converts

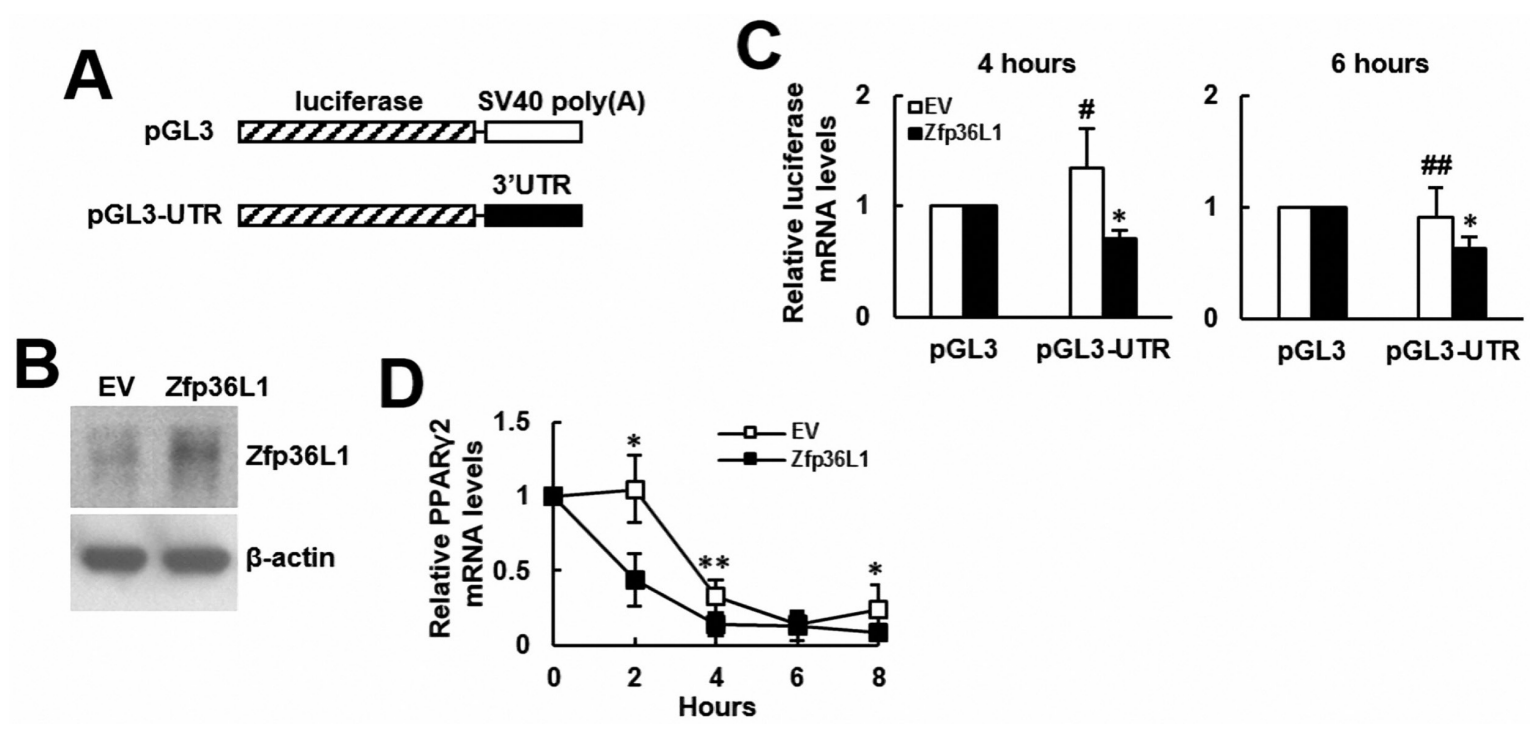

Figure 10: Binding of ZFP36L1-containing protein complexes on the 3'UTR of Ppary 2 mRNA down-regulated gene expression. A. Schematic representation of the pGL3 control and pGL3-UTR constructs. B. Western blot analyses. The ZFP36L1 levels in C3H-EV (EV) and C3H-ZFP36L1 (ZFP36L1) cells were shown. C. RT-qPCR analyses. ZFP36L1-overexpressing (ZFP36L1) and control (EV) C3H10T1/2 cells were transfected with $2 \mathrm{mg}$ of either pGL3-promoter (pGL3) or pGL3-UTR construct along with $0.1 \mathrm{mg}$ of a Renilla luciferase reporter as a transfection control. Cells were harvested 4 and $6 \mathrm{~h}$ post transfection, and the levels of the luciferase transcripts were examined. Luciferase levels were normalized to the levels of Renilla and $\beta$-actin. The normalized luciferase signals of cells receiving pGL3-UTR were compared to the normalized luciferase signals of cells receiving pGL3 (to which a value of 1 was assigned). Data represent the mean \pm S.D. from three analyses. *, $P<0.01$; ${ }^{*}, P=0.479$; ${ }^{\#}, P=0.239$ versus control. D. RT-qPCR analyses. ZFP36L1overexpressing (ZFP36L1) and control (EV) C3H10T1/2 cells were induced to undergo adipogenic differentiation with concomitant treatment of troglitazone $(1 \mu \mathrm{M})$ for $24 \mathrm{~h}$. Cells were then treated with Actinomycin D $(1 \mu \mathrm{g} / \mathrm{ml})$ and were harvested $0,2,4,6$, and $8 \mathrm{~h}$ post treatment. The relative expression levels of Ppary 2 mRNA were calculated in relation to the level at $0 \mathrm{~h}$ (to which a value of 1 was assigned). Data represent the mean \pm S.D. from three experiments. ${ }^{*}, P<0.05 ;^{* *}, P<0.01$ versus corresponding EV control. 
osteoblastic cells to adipocytes [15]. Partially knockout of Ppary enhances bone formation in transgenic mice through increased osteoblastogenesis [16]. With these evidences in mind, our data, which showed that ZFP36L1 overexpression decreased Ppar 2 mRNA expression, whereas ZFP36L1 knockdown increased Ppar 2 mRNA expression and decreased osteoblastic differentiation, and that TZD prevented ZFP36L1 from inhibiting adipogenic differentiation, support the speculation that downregulation of PPAR $\gamma 2$ expression could be a critical step for ZFP36L1 to repress adipogenic lineage but to promote osteoblastic lineage. Moreover, by showing that (i) ZFP36L1 overexpression decreased PPAR $\gamma 2$ expression without concomitantly decreasing Ppary promoter activity (Figure 7), (ii) endogenous ZFP36L1 interacted with Ppary 2 mRNA and bound its 3'UTR (Figure 8), and (iii) ZFP36L1 overexpression inhibited the expression of a luciferase transcript containing the 3'UTR of Ppary2 mRNA, and enhanced the degradation of Ppar 2 mRNA (Figure 10), our data suggest that ZFP36L1 regulates adipogenic differentiation at post-transcriptional level, and that ZFP36L1 might interact with the 3'UTR of Ppary2 mRNA, presumably by binding to the AU-rich element(s), and mediate its degradation.

On the other hand, it is noteworthy that Runx2 can also repress osteoblastic differentiation once recruiting co-repressors. For examples, HDACs such as HDAC3 and HDAC7 bind to Runx2 and prevent it from transactivating the expression of osteocalcin and osteopontin, respectively $[17,18]$. Interestingly, we found that ZFP36L1 overexpression in C3H10T1/2 cells decreased the expression of Hdac3 and Hdac7 mRNAs during osteoblastic differentiation (Supplemental Figure S2), which supports, in part, the possibility that ZFP36L1 might be able to decrease the expression of HDACs to release Runx2's transcriptional activity from the inhibitory HDACs' effect.

While our studies focused on the role of ZFP36L1 in aging-related bone loss, the finding that ZFP36L1 was able to regulate adipogenesis raises a question as to whether this molecule may also play a potential role in obesity. Several microRNAs have been found to enhance or repress adipogenesis of preadipocytes, MSCs, obese adipose tissues, and obese adipocytes depending on their targets [20, and the references therein]. Recently, Di Bernardo et al. reported that the sera of overweight individuals was able to promote the adipogenic differentiation of bmMSCs while partially repress osteogenesis [21]. Therefore, it will be of interest to examine if ZFP36L1 is involved in the mechanisms by which microRNAs and the circulating factors regulate adipogenesis and obesity. Besides, ZFP36L1 has also been shown to degrade the transcripts of numerous senescenceassociated secretory phenotype (SASP) components which otherwise enhance senescence [22], which indicates a regulatory role of Zfp36L1 in senescence. Accordingly, we overexpressed Zfp36L1 in the bmMSCs derived from aged rats (Supplemental Figure S3A), and stained the cells for the senescence-associated $\beta$-galactosidase. The results showed that Zfp36L1 overexpression decreased the number of stained cells (Supplemental Figure S3B). We also examined the expression of Cdkn1a and Cdkn2a (cell cycle inhibitors), Rb1, and Rb2. Our data showed that ZFP36L1 overexpression decreased the expression of Cdkn1a, Cdkn2a, and Rb2 mRNAs, but increased the expression of Rb1 mRNAs (Supplemental Figure S3C). It has been reported by Galderisi et al. that $\mathrm{Rb} 1$ expression in MSCs decreased during in vitro cultivation, and that Rb2/ P130 may play a role in triggering the senescence process in MSCs [23]. Therefore, our data support the notion that down-regulation of Zfp36L1 may be a contributor to the development of senescence phenotype in bmMSCs as well.

In summary, we have shown the aging-associated expression pattern of ZFP36L1, and conducted in vitro and ex vivo experiments to address ZFP36L1's candidacy as an enhancer of osteoblastic differentiation but a repressor of adipogenic differentiation of multipotent cells. These findings, together with the finding that ZFP36L1 was able to bind to the 3'UTR and target Ppary2 mRNA for down-regulation, link this RNA-binding protein to an aging phenotype (bone loss), and support the notion that decreased ZFP36L1 expression in bmMSCs might contribute to aging-related bone loss. Since that Zfp36l1 knockout is embryonically lethal, it would be necessary to establish mouse models allowing for conditional knockout of ZFP36L1 in bones to verify the role of ZFP36L1 in regulating bone formation in aging. Beyond this, our data provide an experimental basis to further delineate the functional significance of ZFP36L1 in aging.

\section{MATERIALS AND METHODS}

\section{Plasmids}

Full-length mouse Zfp36l1 cDNA was cloned into pcDNA3.1(-) vector (Invitrogen, CA, USA) to generate pcDNA3.1-ZFP36L1 for ZFP36L1 overexpression in $\mathrm{C} 3 \mathrm{H} 10 \mathrm{~T} 1 / 2$ cells. The cDNA was also cloned into pLAS2w.Pneo vector to generate pLAS2w-ZFP36L1 for Lentivirus preparation. pLAS2w.Pneo and pshRNAZFP36L1 (a plasmid harboring shRNA targeting Zfp36l1 mRNA for degradation) were purchased from the National RNAi Core Facility at Academia Sinica, Taiwan. A cDNA fragment encoding a 3-repeat-FLAG epitope was cloned 5' to the Zfp36l1 cDNA to generate pcDNA3.13xFLAG-ZFP36L1 which expresses FLAG-tagged ZFP36L1 proteins. pGL3-Ppary-luc, a luciferase reporter driven by Ppary promoter was constructed as described previously [4]. 


\section{Lentivirus preparation and infection}

For ZFP36L1 overexpression or knockdown in MC3T3-E1 cells, pLAS2w-ZFP36L1 or pshRNAZFP36L1 was cotransfected with gag-pol and VSV-G-expressing plasmids into 293T cells for the virus preparation as described previously [4]. Cells were infected with virus $(\mathrm{MOI}=20)$ for $24 \mathrm{~h}$ in the presence of polybrene $(8 \mu \mathrm{g} / \mathrm{ml})$. Infected cells were selected with $2 \mu \mathrm{g} / \mathrm{ml}$ of puromycin (Enzo Life Sciences, Switzerland). Puromycinresistent cells were pooled for experiments. For ZFP36L1 knockdown in C3H10T1/2 cells, infected cells were selected with $5 \mu \mathrm{g} / \mathrm{ml}$ of puromycin. Clone 6 which expressed the lowest level of Zfp36ll mRNA was selected for experiments.

\section{Cell culture}

C3H10T1/2, MC3T3-E1, and 3T3-L1 cells were purchased from American Type Culture Collection. C3H10T1/2 and 3T3-L1 cells were maintained in DMEM (GIBCO-BRL, CA, USA), whereas MC3T3-E1 cells were maintained in MEM $\alpha$ (catalog no. A1049001, GIBCOBRL, CA, USA). Culture media were supplemented with $10 \%$ fetal bovine serum, glutamine, penicillin, and streptomycin. Cells were cultured at $37^{\circ} \mathrm{C}$ in a humidified atmosphere containing 5\% CO2. $\mathrm{C} 3 \mathrm{H} 10 \mathrm{~T} 1 / 2$ cells were transfected with pcDNA3.1-ZFP36L1 and selected with G418 $(1 \mathrm{mg} / \mathrm{ml})$. Twenty two antibiotic-resistant clones were harvested and examined for ZFP36L1 overexpression. Clones 2 and 21 which expressed high level of ZFP36L1 were selected for experiments. The femurs and bone marrow mesenchymal stem cells isolated from the femurs of adult and aged Fisher 344 rats were kind gifts from Dr. Chun-Chin Liang. For the isolation of RNA from rat femurs, the femurs were cryogenically pulverized at $-195^{\circ} \mathrm{C}$ by liquid $\mathrm{N} 2$ using a SPEX 6770 Freezer/Mill (SPEX SamplePrep, NJ, USA). Total RNA was then extracted using Trizol Reagent (Life Technology, MD, USA).

\section{Induction of osteoblastic and adipogenic differentiation}

Osteoblastic induction on MC3T3-E1 and C3H10T1/2 cells, and adipogenic induction on 3T3L1 and C3H10T1/2 cells were performed as described previously [4]. At the end of experiments, for osteoblastic differentiation, cells were fixed and stained with $2 \%$ Alizarin Red S solution (Sigma-Aldrich, MO, USA) for $30 \mathrm{~min}$ at room temperature, and were de-stained with freshly prepared $10 \%$ Cetylpyridinium chloride (CPC) (Sigma-Aldrich, MO, USA) solution for 1 hour at room temperature with gentle rocking. The $\mathrm{CPC}$ solutions were then collected for the measurement of absorbance at 595 $\mathrm{nm}$. For adipogenic differentiation, cells were fixed and stained with $0.3 \%$ Oil Red O (Sigma-Aldrich, MO, USA), and were de-stained with isopropanol for the measurement of absorbance at $510 \mathrm{~nm}$.

\section{Quantitative real-time PCR (RT-qPCR) and western blot analyses}

Total RNA isolation, RT-qPCR analyses, and Western blot analyses were performed as described previously [4]. The 5' and 3' primers used were as follows: mouse Zfp36l1, CCCGATGGCACCAATAACC and GCCCCATGCTAGGAGCAA; mouse Runx2, CTCCGCTGTGAAAAACC and TGAAACTCTTGCCTCGTCC; mouse osteopontin, CCATCTCAGAAGCAGAATCTCC and ATGGTCATCATCGTCGTCC; mouse osteocalcin, TCTCTCTGACCTCACAGATCCC and TACCTTATTGCCCTCCTGCTTG; mouse Hdac3, CAGAGAGTCAGCCCCACCAA and GTAGACCACCGGCCCAGTT; mouse Hdac4, GGGAATGTACGACGCCAAAG and GCTGCATGCGGAGTCTGTAA; mouse Hdac7, GCTGGGTGGCTACCATGTTT and CTGAGGTTGGGTTTCTGTTTCC; mouse Ppary 2, TCGCTGATGCACTGCCTATG and GAGAGGTCCACAGAGCTGATT; mouse $a P 2, \quad$ AAGAGAAAACGAGATGGTGACAA and CTTGTGGAAGTCACGCCTTT; mouse adiponectin, AGCCGCTTATATGTATCGCTCA and TGCCGTCATAATGATTCTGTTGG and mouse $\beta$-actin, CCCTGGCACCCAGCAC and GCCGATCCACACGGAGTAC. The antibodies used were anti-ZFP36L1 (Santa Cruz, CA, USA) and anti- $\beta$ actin (BD Biosciences, CA, USA).

\section{Ex vivo experiments and histological analysis}

Cells were seeded into gelatin/EDC scaffolds and implanted subcutaneously into the back of male nude mice (8 weeks old) as described previously [4]. Implants were retrieved two weeks and one month later for the examination of adipogenic and osteoblastic differentiation by histological analysis, respectively. Histological sections were prepared from each implant and stained with either Oil Red O or Alizarin Red S and DAPI.

\section{Luciferase assays}

To examine the influence of ZFP36L1 overexpression on the activity of Ppary promoter, ZFP36L1-overexpressing and control C3H10T1/2 cells (1 $\mathrm{x} 10^{6}$ ) were transfected with $8 \mu \mathrm{g}$ pGL3-Ppary-luc together 
with a Renilla luciferase reporter $(0.1 \mu \mathrm{g})$ as a normalizer. Cells were induced to undergo adipogenic differentiation, and were harvested at the times indicated for luciferase assays using the Dual-Luciferase Reporter Assay System (Promega, WI, USA).

\section{Preparation of biotin-labeled RNA transcripts and pull-down assays}

Total RNA prepared from C3H10T1/2 cells was used to generate the 3'UTR of Ppary2 cDNA. A T7 RNA polymerase promoter sequence [19] was placed 5' to the cDNA. The 5' and 3' primers used were as follows: (T7)CAGGAAAGTCCCACCCGC and AATTTTATAATGTGGTAATTTTTAAT. PCRamplified products were purified. In vitro transcription was performed to generate biotin-labeled and unlabeled transcripts using the MegaScript T7 kit (ThermoFisher Scientific, MA, USA) following the manufacturer's instruction. The transcripts were purified, and $1 \mu \mathrm{g}$ of biotin-labeled transcript was incubated with $150 \mu \mathrm{g}$ of cytoplasmic lysates for $30 \mathrm{~min}$ at room temperature. The mixtures were then mixed with streptavidin-coated magnetic Dynabeads (ThermoFisher Scientific, MA, USA) to collect the biotin-linked ribonucleoprotein complexes. Western blot analyses were then performed to identify the protein component of the complexes.

\section{RNA electrophoretic mobility-shift assays (RNA EMSA)}

Four-microgram aliquots of cytoplasmic fractions were incubated with $0.5 \mathrm{nM}$ of biotin-labeled RNA probes and with certain amounts of unlabeled probes (for competition experiments) for $30 \mathrm{~min}$ at room temperature in a $20-\mu 1$ mixture. The mixtures were electrophoresed through $4 \%$ nondenaturing polyacrylamide gels, and electrotransferred onto positively charged nylon membranes. Protein-RNA crosslink on the membranes was performed by exposing the membranes to UV using a Stratalinker (Stratagene, USA). The membranes were incubated with streptavidin-HRP $(0.1 \mu \mathrm{g} / \mathrm{ml})$, and the signals were visualized by the method as described for Western blot analyses.

\section{Immunoprecipitation of ribonucleoprotein complexes}

Examination of the putative binding of ZFP36L1containing protein complexes on Ppar 2 mRNA was performed as described previously [19]. Briefly, ZFP36L1overexpressing and control cells were mixed with equal volume of polysome lysis buffer plus inhibitors of RNases and proteases. The mixtures were centrifuged and the pellets were discarded. The supernatants were mixed for $16 \mathrm{~h}$ at $4^{\circ} \mathrm{C}$ with protein $\mathrm{A}$ beads pre-coated with either anti-ZFP36L1 or anti-IgG antibody. The beads were then washed with NT-2 buffer, and the RNAs were isolated from the precipitated ribonucleoprotein complexes for RT-qPCR analyses.

\section{Statistical analysis}

Statistical difference was determined using Student's $t$ test. Paired $t$ test was used in ex vivo experiments.

\section{ACKNOWLEDGMENTS}

We would like to thank Dr. Shau-Ku Huang for the critical reading of this manuscript, and Ms. Hsiang-Yun Tang for technical assistance.

\section{CONFLICTS OF INTEREST}

There is no conflict of interest.

\section{REFERENCES}

1. Verma S, Rajaratnam J, Denton J, Hoyland J, Byers R. Adipocytic proportion of bone marrow is inversely related to bone formation in osteoporosis. J. Clin. Pathol. 2002; 55: 693-698.

2. Rosen C, Bouxsien M. Mechanisms of disease: Is osteoporosis the obesity of bone? Nat. Clin. Pract. Rheumatol. 2006; 2; 35-43.

3. Liang CT, Barnes J, Seedor H, Quartuccio HA, Bolander M, Jeffrey JJ, Rodan GA. Impaired bone activity in aged rats: Alterations at the cellular and molecular levels. Bone. 1992; 13: 435-441.

4. Tseng K-Y, Lin S. Zinc finger factor 521 enhances adipogenic differentiation of mouse multipotent cells and human bone marrow mesenchymal stem cells. Oncotarget. 2015; 6:14874-14884. doi: 10.18632/oncotarget.3900.

5. Blackshear PJ. Tristetraprolin and other $\mathrm{CCCH}$ tandem zinc-finger proteins in the regulation of mRNA turnover. Biochem. Soc. Trans. 2002; 30: 945-952.

6. Baou M, Jewell A, Murphy, JJ. TIS11 family proteins and their roles in posttranscriptional gene regulation. J. Biomed. Biotechnol. 2009; 2009:634520.

7. Lai WS, Blackshear PJ. Interactions of $\mathrm{CCCH}$ zinc finger proteins with mRNA: tristetraprolin-mediated AU-rich element-dependent mRNA degradation can occur in the absence of a poly(A) tail. J. Biol. Chem. 2001; 276:2314423154.

8. Carballo E, Lai WS, Blackshear PJ. Feedback inhibition of macrophage tumor necrosis factor- $\alpha$ production by tristetraprolin. Science 1998; 281:1001-1005.

9. Stumpo DJ, Byrd NA, Phillips RS, Ghosh S, Maronpot 
RR, Castranio T, Meyers EN, Mishina Y, Blackshear PJ. Chorioallantoic fusion defects and embryonic lethality resulting from disruption of $\mathrm{Zfp} 36 \mathrm{~L} 1$, a gene encoding a $\mathrm{CCCH}$ tandem zinc finger protein of the Tristetraprolin family. Mol. Cell. Biol. 2004; 24:6445-6455.

10. Stumpo DJ, Broxmeyer HE, Ward T, Cooper S, Hangoc G, Chung YJ, Shelley WC, Richfield EK, Ray MK, Yoder MC, Aplan PD, Blackshear PJ. Targetted disruption of Zfp36L2, encoding a $\mathrm{CCCH}$ tandem zinc finger RNA-binding protein, results in defective hematopoiesis. Blood 2009; 114:24012410.

11. Sanduja S, Blanco FF, Young LE, Kaza V, Dixon DA. The role of tristetraprolin in cancer and inflammation. Front Biosci. 2012; 17:174-188.

12. Jiang SS, Chen C-H, Tseng K-Y, Tsai F-Y, Wang MJ, Chang I-S, Lin J-L, Lin S. Gene expression profiling suggests a pathological role of human bone marrow-derived mesenchymal stem cells in aging-related skeletal diseases. Aging (Albany NY). 2011; 3: 672-684. doi: 10.18632/ aging. 100355.

13. Reppe S, Olstad OK, Rian E, Gautvik VT, Gautvik KM, Jemtland R. Butyrate response factor 1 is regulated by parathyroid hormone and bone morphogenetic protein-2 in osteoblastic cells. Biochem. Biophy. Res. Commun. 2004; 324:218-223.

14. Sierra J, Villagra A, Paredes R, Cruzat F, Gutierrez S, Javed A, Arriagada G, Olate J, Imschenetzky M, VanWijnen AJ, Lian JB, Stein GS, Stein JL, et al. Regulation of the bonespecific osteocalcin gene by $\mathrm{p} 300$ requires Runx $2 / \mathrm{Cbfa} 1$ and the vitamin D3 receptor but not p300 intrinsic histone acetyltransferase activity. Mol. Cell. Biol. 2003; 23:33393351.

15. Lecka-Czernik B, Gubrij I, Moerman EA, Kajkenova O, Lipschitz DA, Manolagas SC, Jilka RL. Inhibition of Osf2/ Cbfa1 expression and terminal osteoblast differentiation by PPAR-gamma 2. J. Cell Biochem. 1999; 74: 357-371.

16. Akune T, Ohba S, Kamekura S, Yamaguchi M, Chung UI, Kubota N, Terauchi Y, Harada Y, Azuma Y, Nakamura K, Kadowaki T, Kawaguchi H. PPARgamma insufficiency enhances osteogenesis through osteoblast formation from bone marrow progenitors. J. Clin. Invest. 2004; 113: 846855.
17. Schroeder TM, Kahler RA, Westendorf JJ. Histone deacetylase 3 interacts with runx 2 to repress the osteocalcin promoter and regulate osteoblast differentiation. J. Biol. Chem. 2004; 279:41998-42007.

18. Jensen ED, Schroeder TM, Bailey J, Gopalakrishnan R, Westendorf JJ. Histone deacetylase 7 associates with Runx2 and represses its activity during osteoblast maturation in a deacetylation-independent manner. J. Bone Miner. Res. 2008; 23:361-372.

19. Wang MJ, Lin S. A region within the 5'-untranslated region of hypoxia-inducible factor- $1 \alpha$ mRNA mediates its turnover in lung adenocarcinoma cells. J. Biol. Chem. 2009; 284:36500-36510.

20. McGregor RA, Choi MS. microRNAs in the regulation of adipogenesis and obesity. Current Molecular Medicine 2011; 11:304-316.

21. Di Bernardo G, Messina G, Capasso S, Del Gaudio S, Cipollaro M, Peluso G, Casale F, Monda M, Galderisi U. Sera of overweight people promote in vitro adipocyte differentiation of bone marrow stromal cells. Stem Cell Res. \& Therapy 2014; 5:4.

22. Herranz N, Gallage S, Mellone M, Wuestefeld T, Klotz S, Hanley CJ, Raguz S, Acosta JC, Innes AJ, Banito A, Georgilis A, Montoya A, Wolter K, et al. mTOR regulates MAPKAPK2 translation to control the senescenceassociated secretory phenotype. Nat. Cell Biol. 2015; 17:1205-1217.

23. Galderisi U, Helmbold H, Squillaro T, Alessio N, Komm $\mathrm{N}$, Khadang B, Cipollaro M, Bohn W, Giordano A. In vitro senescence rat mesenchymal stem cells is accompanied by downregulation of stemness-related and DNA repair genes. Stem Cells Dev. 2009; 18:1033-1042. 